\title{
Modified Extended External Fixator Frame for Leg Elevation in Trauma
}

\author{
Venkatesh Rajaram Sawant ${ }^{1, \odot ~ C h a n d r a k a n t ~ R a m b h a u ~ G h a r w a d e ~}{ }^{1}$ \\ ${ }^{1}$ Department of Plastic Surgery, Grant Medical College and Sir J \\ Group of Hospitals, Mumbai, Maharashtra, India

\begin{abstract}
Address for correspondence Venkatesh Rajaram Sawant, MCh (Plastic and Reconstructive Surgery), Department of Plastic Surgery, Grant Government Medical College and Sir J Group of Hospitals, Byculla, Mumbai 400008, Maharashtra, India

(e-mail: reconvenky@gmail.com).
\end{abstract}

\begin{abstract}
Keywords

- equinus contracture

- heel ulceration

- limb elevation

- modification of external fixator frame

Lower extremity trauma is very often managed by applying an external fixator to stabilize the fracture site. These limbs very frequently require multiple surgeries, which predisposes them to problems like heel pressure sore, equinus deformity and limb edema. These complications prolong the healing time and create obstacles in rehabilitation. Our aim was to modify the usual uniplanar external fixator in such a way that it is able to provide stable limb elevation and also keep the leg and the foot in such a position, so as to avoid the above-mentioned complications. We used our frame in 10 patients and none of our patients developed heel ulceration or equinus deformity. We found that the frame enables easier daily examination of the wound and improves patient comfort during dressing changes.
\end{abstract}

\section{Introduction}

We have modified the conventional uniplanar external fixator, used to stabilize the fracture site,,$^{1,2}$ to provide limb elevation and keep the foot in proper position, so that complications such as heel pressure sore, ${ }^{2}$ limb edema, ${ }^{3}$ and equinus deformity ${ }^{4}$ can be prevented.

\section{Technique of Application}

The modified frame can be easily attached to any external fixator by adding extra rods and pins. Other than that, it does not require any special hardware or equipment.

It consists of two rods (A and B) attached at an angle to one of the pins of the external fixator angle, so that the rods span across the foot comfortably, as shown in - Fig. 1 . These two rods are now connected with a horizontal rod (C), which is positioned at the level of the metatarsal head and adjusted to keep the foot in neutral or slightly dorsiflexed position in order to prevent equinus deformity.

Two extended, extra long vertical rods ( $D$ and $E$ ) are attached to this horizontal rod C ( - Fig. 2). This will enable us to give limb elevation. A second horizontal rod (F) is attached lower down, which will give this frame stability. The frame is now adjusted to give appropriate limb elevation. Keep a pillow under the knee for supporting and comforting the patient. The patient is made to wear a slipper over the affected foot to prevent the possibility of a pressure sore over the metatarsal head.

\section{Results}

We have used this frame for 10 patients over a period of 6 months from September 2018 to February 2019. All patients were cases of lower limb fractures that had external fixation done and required plastic surgical intervention.The modified frame was applied as discussed above.

\section{Lower $1 / 3$ fractures -4 . \\ Middle $1 / 3$ fractures -4 . \\ Upper $1 / 3$ fractures -2 .}

The average duration of stay for these patients was approximately 2 months. All patients have undergone flap cover. There were no incidences of heel ulcerations or equinus contractures in any of these patients

\section{Discussion}

In most cases of lower limb trauma, the fracture site is stabilized by an external fixator. ${ }^{1}$ These patients are prone to several issues which result in poor healing if not addressed. ${ }^{1-3}$

Limb edema ${ }^{3}$ is frequently observed in these patients, which often persists due to inflammation, infection, or multiple other causes like lack of proper limb elevation.

Heel ulcers ${ }^{2}$ are frequently seen due to the improper positioning, which results in pressure over the heel.
License terms

(a) (1) $\ominus \circledast$ 


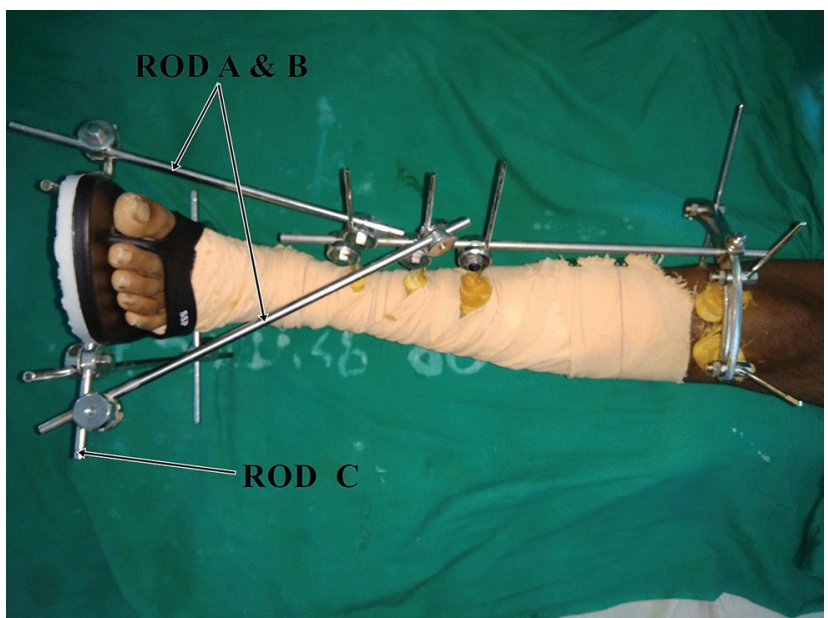

Fig. 1 Top view.

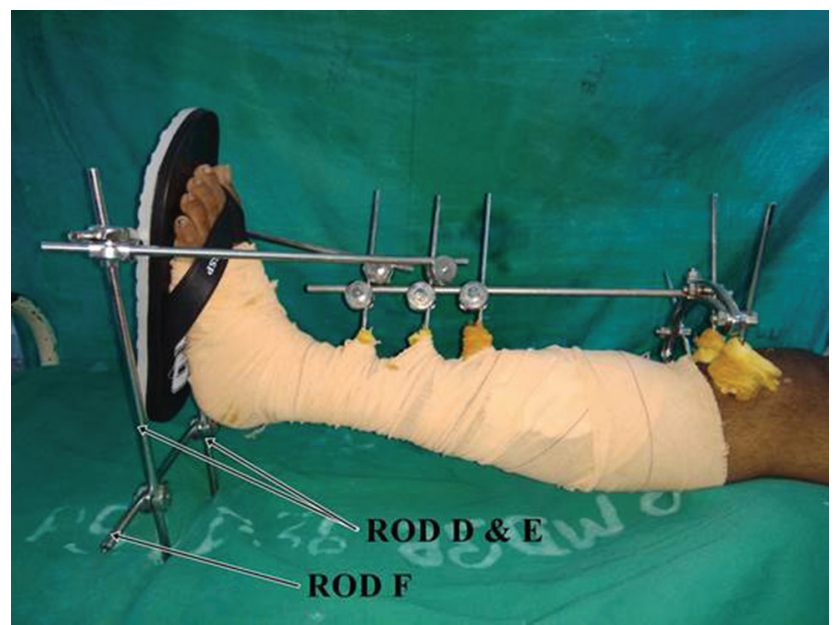

Fig. 2 Side view.

Equinus contractures ${ }^{4}$ develop in patients whose external fixator does not span the ankle joint due to malpositioning of the foot in plantar flexion, especially in severe injuries.

There are also problems with wound inspection and dressing, as plaster of Paris slabs are used to maintain foot in position. These slabs frequently lose their strength due to spillage of wound discharge, which makes the slab soften and break, ${ }^{5}$ failing to maintain the foot in dorsiflexion.

The application of the modified frame has certain advantages.

1. It provides stable limb elevation, which can be calibrated by the surgeon by adjusting the position of the horizontal $\operatorname{rods}(\operatorname{rod} C)$ on the vertical rods (rods $D$ and $E$ ).

2. It maintains the foot in a proper position and prevents the development equinus contracture.

3. It obviates the need for a plaster of Paris slab, so it allows easier dressing changes, which are more comfortable for the patient. It also requires less personnel for change of dressings and allows easier circumferential examination of the wound.

4. The frame is very easy to assemble and can be easily done bedside.
5. We have also found it useful in certain local flaps like reverse sural flap to offload the donor area, so the patient can still remain supine rather than turning prone or lateral to prevent pressure on the pedicle.

We have noted certain drawbacks after applying the frame.

1. Some patients developed knee pain due to the hyperextension at the joint, so we placed a pillow under the knee for support.

2. We have also observed that some patients developed erythema over the foot at the level of rod C, for which we made them wear a slipper over the foot to relieve the pressure; alternatively, soft padding can also be given over the rod.

3. The frame cannot be assembled in patients who have a ring fixator.

Our frame has the advantage of not having to pass any pins through any bone. This decreases pain, discomfort, and overall morbidity. This is unlike the method described by Budford et al. ${ }^{6}$

The rod at the level of the metatarsal head prevents the development of equinus contracture, which is not done in the method by Castro-Aragorn ${ }^{2}$ et al or Sawyer et $\mathrm{al}^{7}$ and Berkowitz ${ }^{1}$ et al.

\section{Conclusion}

The modified frame is easy to apply and provided stable and reliable limb elevation. It reduced the incidence of heel ulceration and equinus deformity. The overall convenience of daily examination of the leg and dressing changes was also greatly improved.

\section{Conflicts of Interest}

None declared.

\section{References}

1 Berkowitz MJ, Kim DH. Using an external fixation "kickstand" to prevent soft-tissue complications and facilitate wound management in traumatized extremities. Am J Orthop 2008;37(3):162-164

2 Castro-Aragon OE, Rapley JH, Trevino SG. The use of a kickstand modification for the prevention of heel decubitus ulcers in trauma patients with lower extremity external fixation. J Orthop Trauma 2009;23(2):145-147

3 Lohrmann C, Pache G, Felmerer G, Foeldi E, Schaefer O, Langer M. Posttraumatic edema of the lower extremities: evaluation of the lymphatic vessels with magnetic resonance lymphangiography. J Vasc Surg 2009;49(2):417-423

4 Shu H, Ma B, Kan S, Wang H, Shao H, Watson JT. Treatment of posttraumatic equinus deformity and concomitant soft tissue defects of the heel. J Trauma 2011;71(6):1699-1704

5 Halanski M, Noonan KJ. Cast and splint immobilization: complications. J Am Acad Orthop Surg 2008;16(1):30-40

6 Buford GA, Trzeciak MA. A novel method for lower-extremity immobilization after free-flap reconstruction of posterior heel defects. Plast Reconstr Surg 2003;111(2):821-824

7 Sawyer JR, Kelly DM, Rhodes LN, Beaty JH, Terry Canale S, Warner WC, Jr. Use of a "kickstand" modification for external fixation of lower extremity fractures in children. J Child Orthop 2011;5(1):63-67 